\title{
Ouvrir, suspendre et lever une audience à distance tenue par visio-conférence
}

Effets performatifs des actes de langage et situations équipées

Performative Effects of Speech Acts in Mediated Interaction: Opening and

Closing a Courtroom Trial by Video-Conference

Christian Licoppe

\section{OpenEdition}

Journals

Édition électronique

URL : http://journals.openedition.org/edc/377

DOI : 10.4000/edc.377

ISSN : 2101-0366

Éditeur

Université de Lille

Édition imprimée

Date de publication : 1 décembre 2006

Pagination : $95-117$

ISBN : 2-9514961-7-6

ISSN : $1270-6841$

Référence électronique

Christian Licoppe, "Ouvrir, suspendre et lever une audience à distance tenue par visio-conférence », Études de communication [En ligne], 29 | 2006, mis en ligne le 19 janvier 2009, consulté le 10 décembre 2020. URL : http://journals.openedition.org/edc/377 ; DOI : https://doi.org/10.4000/edc.377

Ce document a été généré automatiquement le 10 décembre 2020.

(c) Tous droits réservés 


\title{
Ouvrir, suspendre et lever une audience à distance tenue par visio-conférence
}

\author{
Effets performatifs des actes de langage et situations équipées \\ Performative Effects of Speech Acts in Mediated Interaction: Opening and \\ Closing a Courtroom Trial by Video-Conference
}

Christian Licoppe

\section{Une audience judiciaire levée sans être ouverte}

1 Pour pallier le faible nombre d'affaires judiciaires et le déficit de magistrat qui s'ensuit dans les tribunaux de la collectivité territoriale de Saint-Pierre et Miquelon, l'administration judiciaire permet depuis 2001 que des magistrats de la Cour d'Appel de Paris puissent présider à distance certaines audiences, au moyen d'un dispositif de visioconférence. Dans la configuration la plus usuelle de ces audiences, le ou les juges siègent à Paris ainsi que le greffe, tandis que le procureur, les particuliers concernés et le public se tiennent à Saint Pierre et Miquelon. Selon les cas, les avocats peuvent être présents sur l'un ou l'autre site.

2 J'ai eu l'occasion d'observer une quinzaine d'audiences à distance dans le cadre de cette enquête ${ }^{1}$. Certaines ont donné à voir des particularités inhabituelles dans la manière de gérer l'ouverture ou la levée de l'audience. Dans l'une d'entre elles, le président a levé l'audience sans l'avoir auparavant ouverte en prononçant la formule consacrée («je déclare l'audience ouverte»). Dans quelques autres, la décision est rendue et les participants dispersent leur attention et se mettent à discuter de leurs affaires ordinaires (demandes de nouvelles, arrangements, prises de rendez vous) avant que l'audience ait formellement été levée. Dans l'une d'entre elles, le président ne prend même pas le soin de le faire. Comme si l'énonciation des actes de langage rituels par lesquels le président 
ouvre et lève l'audience apparaissait rétrospectivement moins efficace et même moins nécessaire dans le cas des audiences à distance.

Ces observations ne relèvent pas d'un simple relâchement du formalisme. Les magistrats impliqués souhaitent au contraire montrer à l'administration judiciaire que l'audience à distance est une forme d'audience convenable et appropriée et qu'elle ne conduit pas à des «audiences au rabais » comme le prétendent leurs critiques. D'autre part, il leur importe de ne pas donner l'impression d'une audience bâclée par rapport à ce qui se fait en métropole, ce qui serait immédiatement interprété par les participants locaux comme un nouvel exemple de la condescendance dont ils accusent la France de faire preuve vis-àvis d'eux. Les phénomènes décrits ci-dessus ne sont en fait pas explicités par les magistrats, non pas qu'ils soient délibérément tus ou minimisés, mais parce qu'ils se produisent dans le flux de l'activité, sans laisser d'impression assez précises et durables pour être généralisés.

4 La sociologie pragmatique a montré l'intérêt d'étudier les formes de coordination, et tout particulièrement les points de transition entre les séquences d'activité. Cela permet de rendre compte de deux propriétés de l'action: sa "rigidité", qui se manifeste par des discontinuités plus ou moins grandes dans les scènes traversées par les personnes, et sa complexité, liée à la pluralité des régimes d'action qui dont susceptibles de coexister et de rendre l'action plus susceptible au surgissement de perturbations (Dodier, 1993). L'audience à distance serait alors caractérisée par une moins grande rigidité et une plus grande complexité que les audiences en prétoire, au niveau des phases d'ouverture et de clôture. Je vais explorer cette idée, et tenter d'élucider ainsi un aspect moins étudié de la performativité.

\section{La force performative des actes de langage}

«L'audience est ouverte », «l'audience est levée », « l'audience est suspendue » sont les formules conventionnelles utilisées par les magistrats pour ouvrir une audience judiciaire. Elles s'apparentent étroitement aux exemples que John Austin utilise pour introduire la notion d'actes de langage : « oui » (je prends cette femme pour épouse), «Je baptise ce bateau le Queen Elisabeth", "Je donne et lègue cette montre à mon frère " (Austin, 1970). Tous ces énoncés constituent des actes de langage explicites (la problématique d'Austin se déplaçant ensuite vers une théorie de la force performative du langage en général, dont les énoncés performatifs explicites ne constituent qu'un cas particulièrement pur), puis vers une théorie linguistique de l'action (Laugier, 2004). D'autre part parce que, à la différence du dernier des quatre exemples initiaux d'Austin ( "Je vous parie six pence qu'il pleuvra demain») et de beaucoup de ses exemples ultérieurs, ils réfèrent plus directement à des activités institutionnelles et à des situations de parole publique.

6 Lorsque ce type d'actes de langage est accompli avec bonheur, il contribue à des changements de grande ampleur. Il inaugure un monde dont il contribue publiquement à fixer certaines caractéristiques pour un grand nombre de participants (baptiser un bateau, prendre pour époux) ou instaure un type d'activité très différente de ce qui précède, et vers lequel ceux-ci doivent désormais s'orienter (ouvrir une audience, demander un temps mort dans un match, etc.). La portée de ces actes de langage dépasse même le public co-présent. Ils peuvent agir à distance, puisqu'ils sont en général associés à des pratiques d'inscription, à des écritures «officielles» ou authentifiées. Celles-ci 
fixent la mémoire de leur accomplissement et relayent leurs conséquences bien au-delà du moment de leur énonciation et du public initial ${ }^{2}$.

7 L'analyse de ces actes institutionnels est redevable de deux approches possibles. Une approche pragmatique et formelle, consiste comme chez Austin à déterminer les conditions de félicité dont la complétude est nécessaire pour que l'énoncé puisse prétendre constituer un acte de langage et revendiquer une force illocutoire. Il faut ensuite vérifier que l'acte de langage est bien traité comme tel, c'est-à-dire que les réactions du public (ce que Austin appelle "uptake ») sont compatibles avec ses visées illocutoires et perlocutoires. Tout en restant dans le cadre d'une sociologie formelle, l'analyse de conversation (AC) pointe les limites de l'approche analytique, centrée sur l'énoncé isolé plus que sur la manière dont celui-ci s'inscrit dans un contexte que son énonciation contribue à renouveler. L'AC s'intéresse à l'analyse systématique des méthodes permettant de produire un tel acte de langage de manière reconnaissable et séquentiellement pertinente. Elle montre par exemple que la notion d'acte de langage indirect est mieux comprise si on l'interprète comme la première partie d'un certain type de paire adjacente, les pré-séquences (Levinson, 1983).

8 Vus sous cet angle, les problèmes relevés lors des audiences à distance semblent relever de propriétés spécifiques des actes de langage. Il ne s'agit ni de ratés («misfires») ni d'abus («abuse»). Les actions des participants s'orientent par rapport à l'accomplissement des actes de langage habituels. Ceux-ci sont prononcés, comme en prétoire, par le magistrat qui préside l'audience, c'est-à-dire la personne autorisée et investie de l'autorité nécessaire. C'est plutôt du côté de la cohérence entre leur visée performative et le contexte dans lequel ils sont produits, et des ressources et méthodes dont disposent les acteurs pour l'accomplir, qu'il faut chercher l'origine de certaines particularités des audiences à distance.

\section{Ouvrir une audience en co-présence, dans un prétoire}

L'ouverture d'une audience en prétoire doit résoudre un problème récurrent dans les réunions publiques. Elle doit accomplir de manière économique et efficace une transition entre une situation où la multitude des participants est fragmentée en petits groupes ou en individus à l'attention dispersée et engagés dans des activités différentes (par exemple de multiples conversations séparées) et une situation où d'une part l'attention de tous les participants est focalisée sur une même séquence d'activité, et où d'autre part cette activité est immédiatement intelligible et reconnaissable comme une audience, et une audience du type attendu (Relieu et Brock, 1995). Dans le format canonique d'ouverture de l'audience, ceci est accompli par deux paires d'actions adjacentes.

La première est inaugurée par une sommation, une sonnerie qui retentit, ou un officier de justice qui annonce à forte et haute voix l'arrivée de la cour (« Mesdames et Messieurs, la Cour »). Cette sommation est configurée de manière à projeter comme réponse pertinente un comportement commun (interrompre ses activités en cours et se lever). D'une part les co-présents s'attendent à ce qu'un événement de ce type arrive, d'autre part il est perceptivement assez notable (par son volume sonore) pour manifester son caractère interruptif par rapport aux activités en cours, et enfin les participants moins familiers avec les audiences peuvent s'aligner sur le comportement des avocats, des officiers de police ou des membres du public les plus expérimentés pour produire une réponse pertinente (Drew et Atkinson, 1979). 
11 L'ouverture de l'audience est alors accomplie par une nouvelle paire d'actions. Dans un même mouvement, le ou les magistrats rentrent, s'assoient, et celui qui préside l'audience déclare usuellement «l'audience est ouverte, vous pouvez vous asseoir ». L'énonciation d'une telle formule performative constitue une manière particulièrement efficace d'ouvrir la situation de parole publique, en rendant l'activité dans laquelle elle s'inscrit immédiatement intelligible pour un public qui peut être nombreux et très hétérogène quant à son degré de familiarité avec ce type de situation. La situation installée par le performatif est une audience judiciaire et tout ce qui s'ensuit pourra être évalué par rapport aux critères qui définissent normativement cette activité : le fait que le président de l'audience « tienne » l'audience, que s'y exerce un régime asymétrique d'allocation des tours de parole très différent de celui de la conversation ordinaire, que le type d'action qui s'y déroule relève de catégories particulières (on y comparait, on y plaide dans le cadre d'un débat contradictoire, on y délibère), etc.

Ce déroulement des débuts d'audience en prétoire est quasi-rituel (Garapon, 2001). Il est reproduit à l'identique dans la grande majorité des audiences. Il apparait peu vulnérable à la perturbation: lorsque des variations surviennent, elles seront traitées comme remarquables et effectivement remarquées. L'ouverture d'une audience construit une discontinuité abrupte et nette, au sens où elle est accomplie méthodiquement, rapidement et efficacement, presque indépendamment du nombre de co-présents, et où elle installe une situation de parole publique intelligible comme telle et très différente quant aux modes d'engagement et de régulation interactionnelle qu'elle implique de la situation de co-présence « ordinaire » qui précède.

Un des facteurs qui contribue à cette remarquable économie d'efforts, et en particulier à la félicité de l'acte de langage par lequel le président déclare l'audience ouverte, est le cadrage spatial de la situation. En effet la salle d'audience est construite de manière à assurer une distribution spatiale des rôles, avant même l'ouverture de l'audience. Des emplacements particuliers sont prévus pour le public, pour les parties civiles et les avocats, les prévenus et les officiers de police. Avant l'entrée des juges, les membres de l'audience sont certes dispersés dans des engagements divers, mais leur position dans la salle rend lisible la catégorie pertinente pour décrire leur engagement dans l'activité à venir. En remplissant ce qui apparaît alors une position focale mais vide (le bureau surélevé au fond et au centre de la salle d'audience) l'entrée des juges vient compléter la scène. La paire d'actions que constituent l'acte de langage inaugural du président et l'alignement ostensible de l'attention et des actions du public anime un tableau, intelligible d'un seul coup d'œil comme une audience en prétoire, du fait de la disposition des participants dans un espace cadré et " préparé » pour cette activité.

\section{L'ouverture d'une audience en visio-conférence}

14 Je vais chercher à partir d'un exemple à comprendre et expliciter certains des principes interactionnels par rapport auxquels les participants orientent et évaluent leurs actions, tout particulièrement dans la phase d'ouverture de l'audience à distance. L'audience étudiée ici rend les conclusions du délibéré qui faisait suite à une audience tenue la semaine précédente, avec les mêmes participants, également par visioconférence. Cette audience a pour unique fonction de permettre au président de rendre une décision, et aucune autre affaire ne sera traitée dans ce cadre. 


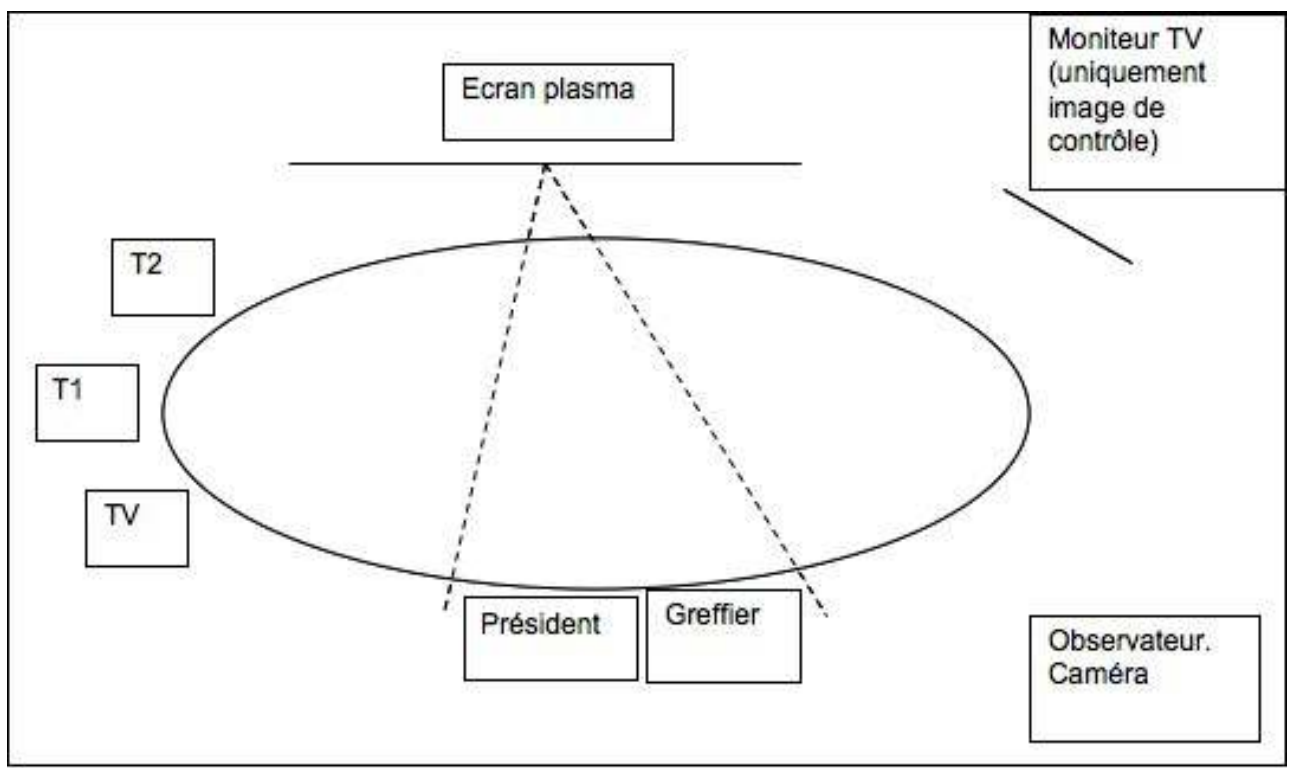

Figure 1 : La salle parisienne. À gauche, hors champ (et donc invisible pour le site distant), le responsable technique (T1), un technicien qu'il forme à l'utilisation du dispositif (T2), et un technicien visiteur d'un autre ressort venu pour se former (TV). À droite, l'observateur (hors champ également). Au centre le président et son greffier. Face à eux un grand écran plasma sur lequel s'affiche l'image représentée dans la figure 2. A droite un moniteur affiche l'image de contrôle (c'est-à-dire l'image transmise) pour aider aux réglages. Le cadrage choisi est figuré en pointillé : durant toute la séquence, la caméra est centrée sur le président et le greffier qui seuls jouent un rôle dans l'audience à suivre.

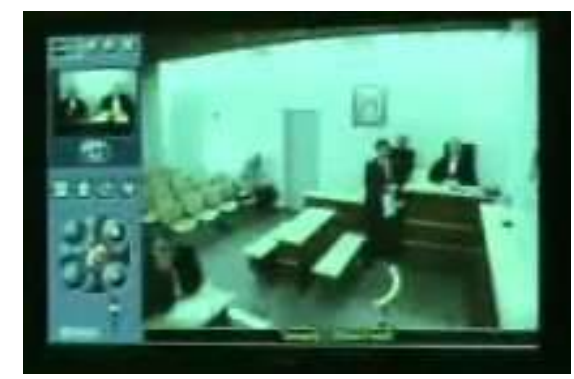

Figure 2 : Image visible du côté parisien à la connexion. Le procureur (en robe) est assis à droite, avec le greffier du tribunal de Saint-Pierre et Miquelon à gauche. Devant eux, debout, le président du Tribunal Supérieur d'Appel. Assis au fond de la première rangée de sièges, la personne dont l'affaire est sur le point d'être traitée. En bas à gauche, un avocat (en robe) qui représente des « témoins assistés » et absents. Dans l'image de contrôle, en haut à gauche, le président et le greffier parisiens. La qualité de l'image présentée ici a été dégradée par rapport à celle effectivement visible à l'écran, pour des raisons de confidentialité.

15 La manière dont les participants sont distribués sur les deux sites est décrite dans les figures 1 et 2 .

Le moment où je commence l'analyse de cette séquence fait suite à un premier essai de connexion où les acteurs se voyaient sans s'entendre. Les participants côté parisiens ont alors convenu de relancer le système, ce qu'ils font avec succès.

\section{Établissement de la connexion audio et vidéo et sommation initiale}

Certaines audiences à distance décalquent presque à l'identique l'organisation séquentielle de la séquence canonique d'ouverture de l'audience en prétoire. Cela arrive pour certaines audiences de première instance, dans des affaires présidées par un juge unique. Tout le tribunal siège à Saint Pierre et Miquelon, à l'exception d'un avocat qui se 
connecte de Paris. Dans ce cas, l'ouverture de l'audience se fait selon une séquence du type :

1. Connexion mutuelle et apparition de l'image et du son des sites distants.

2. Interruption éventuelle des activités en cours et orientation attentionnelle des participants vers les écrans respectifs.

3. le magistrat déclare l'audience ouverte.

4. les participants finissent de se positionner et d'arranger leur posture, puis le magistrat reprend la parole pour annoncer l'affaire.

La comparaison avec l'audience en prétoire montre que dans ce cas, c'est l'apparition instantanée de l'image et du son à la connexion qui joue le rôle de la sommation inaugurale, et invite à l'alignement des participants.

Le moment exact de la connexion étant imprévisible, les participants sont saisis dans l'état où ils sont à cet instant. D'autre part le fait de se lever et s'asseoir pose des problèmes de cadrage. Une convention s'est graduellement développée chez les participants réguliers, selon laquelle ils ne se lèvent plus, ni à ce moment, ni même plus tard, ce qui surprend les magistrats qui viennent pour la première fois. Cela signifie que bon nombre de participants, apparaissent assis à la connexion, et que la réponse appropriée à la sommation que constitue celle-ci, est l'alignement attentionnel des participants vers l'écran.

Divers comportements empiriquement observables témoignent de la manière dont les participants traitent l'établissement de la connexion comme une forme de sommation. Il arrive que des conversations prennent place pendant que le technicien cherche à établir le lien, alors que retentit la sonnerie qui marque la demande de connexion. Les acteurs adoptent alors un débit particulier, rapide et à voix basse. Ils manifestent ainsi le fait que leur échange s'inscrit dans un espace contraint par l'imminence de la connexion. Enfin, pour pallier aux difficultés de démarrage, une liaison téléphonique entre les deux sites est presque toujours ouverte pour permettre aux participants de coordonner leurs opérations. Après la connexion, ces conversations téléphoniques sont très rapidement et spontanément interrompues en un ou deux tours de clôture, les interlocuteurs manifestant ainsi publiquement leur souci mutuel de pouvoir s'engager pleinement vers l'écran le plus rapidement possible.

21 Considérons maintenant ce qui se passe dans notre exemple au moment de la connexion. Les premiers échanges sont orientés vers la ratification publique de la qualité de la connexion («ah voilà ») et plus spécifiquement sa qualité sonore (« on vous entend »). Ils s'orientent rétrospectivement par rapport à la première tentative ratée de connexion pour laquelle le son ne passait pas. Ces tours de parole sont produits simultanément par plusieurs participants sur les deux sites, ce qui rend visible comment chaque participant est également concerné par l'événement perceptif et interactionnel que constitue la mise sous image.

Cette manière de thématiser la manière dont un événement technique soudain (l'établissement de la connexion) crée des opportunités ou des contraintes interactionnelles est caractéristique des "échanges confirmatifs» (De Fornel, 1994). Ceux-ci traduisent une fragilité spécifique à la situation de démarrage de la visioconférence. Contrairement à la sonnerie ou à l'annonce qui résonne dans le prétoire, il faut s'assurer de part et d'autre du caractère partagé de l'événement de sommation. 

l'arrivée des autres participants, pour faciliter les entretiens et les arrangements avant l'audience. Ils se sont rapidement aperçus que cela conduisait à une situation qu'ils jugeaient trop désordonnée. D’un côté les participants saint-pierrais étaient souvent assis, silencieux, et attentifs à l'écran. De l'autre les participants parisiens restaient debout, parlaient entre eux, passaient devant la caméra, etc. Pour remédier à ce désordre et faciliter le travail d'alignement attentionnel, ils en sont venus à ne lancer la connexion qu'une fois les participants (et surtout les magistrats parisiens) installés. Ceci montre comment le fait que les participants traitent l'arrivée de l'image et du son comme une sommation peut être délibérément mis en œuvre à des fins normatives.

\section{Saluer et reconnaître pairs et collègues}

L'établissement de la liaison est instantané. Tous les participants dans le champ des caméras deviennent visibles simultanément. Ils sont en quelque sorte égaux devant l'événement de connexion. Ceci confronte le président de l'audience à plusieurs problèmes pratiques. Comment prendre l'initiative de la parole pour préparer l'installation des modalités interactionnelles caractéristiques d'une audience? Comment prendre en compte les participants qui viennent d'apparaître à la connexion d'une manière ordonnée, sachant qu'ils ne peuvent être reconnus que les uns à la suite des autres, même s'ils sont devenus visibles simultanément ? Comment s'assurer que tous les participants pertinents sont disponibles pour l'audience et bien positionnés par rapport à l'écran?

Une transition entre les échanges confirmatifs et la mise en place de procédures systématiques de résolution de ces problèmes est tout d'abord accomplie. Le procureur de Saint-Pierre et Miquelon adresse une salutation aux participants visibles pour lui de l'autre côté de l'écran (par un petit signe de la main d'acquiescement et un "bonjour " générique, adressé à l'écran, dont le statut est équivoque, entre échange confirmatif (dont la vocation est d'initier une réponse, de qui que ce soit de l'autre côté de l'écran) et initiation d'une séquence de salutations. Le président prend l'initiative de la parole pour saluer nominalement le procureur (ce qui transforme rétrospectivement le bonjour générique de celui-ci en salutation initiale) et enchaîner immédiatement vers une salutation adressée au président du Tribunal Supérieur d'Appel de Saint-Pierre et Miquelon («Bonjour Monsieur le procureur, bonjour Thierry Pillon »).

Cet échange peut être interprété comme le premier d'une série. Ceci contraint la possibilité pour un autre participant de prendre la parole à la place du président, une fois la salutation retournée. Effectivement celui-ci prend à nouveau la parole pour saluer, par son nom, le greffier de Saint-Pierre et Miquelon. Ce dernier ne produit en réponse qu'un hochement de tête différé, puisqu'il survient après que le président ait lui-même enchaîné en s'adressant à un autre participant (indiquant ainsi qu'il n'attendait pas particulièrement de réponse). Les deux interlocuteurs traitent donc cette salutation différemment de la précédente. Elle s'adresse à un greffier et vient juste après un échange de salutations entre magistrats. La succession temporelle des adresses rend visible une hiérarchie relationnelle.

27 Avec le greffier, le président produit un échange de salutation qui tout en marquant une distinction entre la collégialité des magistrats et les relations de collaboration plus hiérarchisées entretenues avec les greffiers, compose également un ensemble : une série 
d'actions collaboratives à travers laquelle sont remarqués et reconnus tous les membres du tribunal saint-pierrais, dans le cadre d'une solidarité juridique des deux cours qui fonde la possibilité et la légitimité de l'activité en cours $^{3}$. La manière ordonnée dont est accomplie cette prise en compte de collègues distants ne se fonde pas sur l'activité en cours mais sur le fait qu'à partir du moment où ils sont présents à l'écran, ils sont disponibles pour être interpellés, et redevables de ce traitement de faveur.

Le président effectue alors une pause plus marquée sans que personne ne parle, ce qui marque à ce stade l'orientation des participants vers un régime d'allocation des tours de parole biaisé en faveur de ce magistrat, si l'on compare aux règles ordinaires d'allocation des tours (Sacks et al., 1974). En régime d'audience, si le président n'a pas sélectionné un locuteur suivant, il est difficile et rare qu'un autre locuteur s'auto-sélectionne pour poursuivre.

\section{«Animer » à l'écran les participants pertinents pour l'activité}

Après cette pause, le président poursuit donc en s'adressant sur un mode encore différent aux autres participants. Il s'adresse à un locuteur explicitement nommé, mais cette fois sans le saluer directement. $\mathrm{Vu}$ son placement séquentiel cette apostrophe doit être comprise et traitée différemment de la série de salutations qui ont précédé. L'avocat est interpellé sous son titre et son nom, et surtout de manière contextualisée, par un constat relatif au fait qu'il est visible à l'écran (« Maitre Condamine, vous êtes visible à l'écran »). D'un côté il s'agit d'«animer" publiquement un personnage visible dans le dispositif, reconnaissable comme professionnel impliqué dans l'activité à venir (ne serait-ce que parce qu'il est en robe). De l'autre ce tour permet de confirmer sa présence sur la scène de cette activité (celle-ci se confondant avec l'écran). Il confère une identité située à la personne à laquelle il est adressé et ratifie comme pertinente sa position. L'avocat réagit par un simple signe de tête, sans se lever.

Le président confirme en enchaînant, s'adressant cette fois vers un autre personnage visible (« et je vois également monsieur Maginel au fond»). Bien qu'aucun titre professionnel ne soit accordé, cette apostrophe est étroitement liée à la précédente, du fait de la présence des deux connecteurs "et » et "également », par l'absence séquentiellement notable de termes de salutation, et par une structure d'unité de tour similaire qui attache la mention du nom de l'intéressé à la possibilité avérée de le voir sur l'écran. L'intéressé réagit à cette interpellation comme l'avocat. Il se lève et se rassoit, manifestant probablement ainsi une forme plus appuyée de déférence (puisqu'il est là en tant que personne privée dont le dossier passe en appel). Il manifeste à son tour sa compréhension du fait que l'adresse du magistrat n'implique pas séquentiellement d'autre réaction de sa part. Avocat et justiciable s'orientent donc tous deux vers le caractère préliminaire des actions du président. Celles-ci sont traitées comme préfaçant l'audience à venir.

Dans le cas étudié ici, puisque les participants sont présents et visibles à l'écran, l'activité du président durant cette phase vise surtout à ratifier leur présence, et, en les nommant explicitement, les engager par rapport à l'audience qui va se tenir. D'autres configurations sont problématiques parce que certains participants ne sont pas visibles à l'écran. C'est le cas dans l'exemple suivant, tiré de l'audience tenue la semaine précédente, avec les mêmes participants, à propos de la même affaire.

$\mathrm{Au}$ commencement, le président nomme les personnes qu'il voit à l'écran comme composant la «salle» (« Oui euh alors bon, de toute façon aujourd'hui à St Pierre nous 
n'avons que Monsieur Magimel euh dans la salle mais il n'y a personne d'autre »). Il rend ainsi public le fait que la scène de l'activité se confond avec l'écran. Ce tour est traité comme une pré-requête à la fois par le procureur qui répond en mentionnant un avocat présent, impliqué, mais pas visible, et par le technicien qui cherche ostensiblement à recadrer. Ne voyant pas encore l'avocat, le président amorce une nouvelle pré-requête dans laquelle l'exigence normative est plus forte puisqu'il explicite le fait que l'avocat n'est toujours pas à l'écran. Le technicien répond finalement en cadrant le participant manquant ce qui suscite l'approbation simultanée du président et du procureur. Le recadrage de la scène de manière à ce que l'écran englobe tous les participants ratifiés, orientés et arrangés de telle manière que leurs segments transactionnels se recouvrent et se chevauchent avec ceux des magistrats parisiens, constitue un accomplissement collaboratif.

D'une manière générale on peut dire que les participants impliqués traitent systématiquement l'évocation de leur nom comme une "pré-requête »", visant indirectement à les faire venir à l'écran. Là, le technicien recadre spontanément de manière à avoir l'avocat mentionné dans le cadre. Dans d'autres cas, les participants euxmêmes se déplacent. Dans une des audiences que nous avons pu observer, le participant qui vient d'être ainsi évoqué passe sa tête de sorte qu'elle devient visible dans le coin de l'écran, à l'amusement des magistrats. Il introduit ainsi une dimension ironique dans sa manière de traiter la pré-requête de repositionnement du président, s'orientant simultanément vers l'exigence normative d'être présent à l'écran, et vers les ressources et les contraintes qu'offrent la situation: «En introduisant ainsi une distance parodique ou ironique à leur activité pratique, les interactants se rendent mutuellement visibles les actions localement situées, les opérations pratiques qui leur sont nécessaires pour réaliser de façon efficace et routinière une interaction visiophonique ordinaire " (De Fornel, 1994). Enfin dans plusieurs cas, les participants anticipent légèrement le tour où ils vont être apostrophés, et se repositionnent d'eux-mêmes pour être plus visibles au moment où ils s'attendent à ce que le président s'adresse à eux.

En traitant les apostrophes produites par le président de l'audience comme une série de pré-requêtes, ils participent à construire un arrangement d'écran qui symétrise leur accès mutuel et rend visible une « communauté de disponibilité » (Kendon, 1992). Ils montrent leur capacité à évaluer leur propre position à l'écran par rapport à cette exigence normative et leur engagement dans la construction d'un cadre interactionnel commun.

\section{Composer un tableau intelligible d'un regard}

Le placement séquentiel de cette série de salutations, apostrophes et évocations n'est pas sans conséquence. En prétoire, les audiences durent plusieurs heures et traitent plusieurs affaires à la suite. Il est donc coutumier pour les magistrats de vérifier et ratifier publiquement la présence et la pertinence des différentes parties, jute avant de traiter l'affaire qui les concerne (donc pendant l'audience). A l'inverse, les audiences à distance traitent le plus souvent une seule affaire. Cette particularité rend possible (mais pas nécessaire) d'effectuer ces vérifications avant l'ouverture formelle de l'audience. Le faire avant l'ouverture permet de régler un problème interactionnel spécifique à cette forme d'audience : l'intelligibilité du tableau présenté à l'écran.

L'établissement de la connexion présente en effet un tableau des participants qui n'est pas nécessairement ordonné. Le problème pratique qui se pose au président et aux 
participants est de parvenir à le réorganiser pour satisfaire deux exigences : s'assurer que tous les participants pertinents sont présents à l'écran, et qu'ils s'y trouvent distribués d'une tel manière que le tableau ainsi composé soit aligné avec l'activité en cours, cohérent avec l'idée qu'on peut se faire de l'audience à venir, et avec la distribution des rôles et des places qu'elle suppose. Dans le cas étudié ici, les participants de Saint-Pierre et Miquelon sont installés dans un prétoire, et y occupent des places compatibles avec une audience en présence ou tout au moins compatibles avec interprétation du tableau composé comme représentant une situation d'audience (le greffier à côté du procureur, le justiciable assis au premier rang). Quand les audiences se tenaient autour d'une simple table de réunion, dans la bibliothèque du Tribunal de Saint Pierre et Miquelon, le travail de mise en intelligibilité pouvait prendre une forme plus complexe et nécessiter des déplacements de la plupart des participants.

Cette exigence d'intelligibilité (" accountability») du tableau composé à l'écran montre la pertinence pratique de deux manières distinctes de s'engager dans l'audience à distance. Dans la première, l'écran est transparent à l'activité. Les participants interagissent «à travers » l'écran, sans même le remarquer tant il est intégré à leur gestion conjointe de la situation. Dans la seconde, un participant appréhende l'écran comme un tableau figuratif. Il se désengage, et devient spectateur d'un écran qu'il saisit comme un objet en soi, un tableau porteur d'un sens qu'il s'agit de déchiffrer, et susceptible de modifications. Cette distinction recoupe la description donnée par Heidegger de la dualité d'une activité équipée. Dans son exemple classique de l'usage du marteau, le marteau est une extension de la main du charpentier (l'utilisateur, son bras et le marteau composent une entité intégrée dans le cadre de l'activité) ${ }^{5}$, jusqu'à ce que le marteau fasse problème (par exemple en se brisant), et que le regard réflexif de l'utilisateur se tourne vers l'instrument plutôt que sur son projet. Abîmé, le marteau se sépare de l'utilisateur et devient une entité distincte dont ce dernier prend en compte les spécificités afin d'y adapter son action.

38 Cette tension entre deux modes de couplage est parfois explicitée publiquement. La force normative de l'exigence d'intelligibilité de l'écran en tant que «tableau » devient alors visible. Cette situation est survenue pour une audience dont le président d'audience était à Paris, entouré par un avocat et une greffière. Le technicien avait spontanément cadré l'image sur ces trois participants. Après la connexion le président a pris l'initiative de préciser à ses interlocuteurs que ce qu'ils voyaient à l'écran ne représentait pas un magistrat entouré de ses deux assesseurs (c'est-à-dire un collège de magistrats), thématisant ainsi directement la possibilité de traiter l'écran comme un tableau. Il s'est même résolu à demander au technicien de corriger le cadre en en faisant sortir temporairement l'avocat. Il a préféré renoncer à l'exigence de visibilité à l'écran des participants concernés pour composer un tableau moins équivoque, et plus aisément lisible par rapport à l'activité à venir.

39 Cet exemple montre deux choses. D'une part les participants expérimentés aux audiences à distance développent une forme de vigilance par rapport au tableau d'écran dans lequel ils sont insérés et aux significations que celui-ci peut projeter, ainsi qu'une compétence réflexive à l'évaluer et la corriger relativement au type d'audience qui est en jeu. D'autre part la possibilité de passer ainsi publiquement d'un mode de couplage à l'autre constitue une ressource pratique pour accomplir une situation ordonnée et intelligible par rapport à l'activité en cours ${ }^{6}$. 


\section{Discussion}

\section{Le problème de la saillance relative des énoncés performatifs d'ouverture}

\section{participants :}

- La possibilité de façonner l'établissement de la connexion audio et vidéo de manière à ce qu'elle puisse apparaître et être traitée collectivement comme une sommation.

- La possibilité pour le président de l'audience de produire, en s'appuyant sur la mobilisation située de différentes structures institutionnelles de relations, une succession ordonnée de salutations et d'apostrophes.

- La possibilité de découpler un engagement interactionnel dans lequel les participants sont focalisés sur l'interaction avec autrui à travers l'écran (alors quasi-transparent) d'une attitude plus circonspecte vis-à-vis de l'image présentée (alors traitée comme un tableau).

Plus la situation est complexe (par exemple lorsqu'il y a de nombreux participants, et que certains d'entre eux sont nouveaux et peu familiers des audiences à distance) et peu lisible (par exemple lorsque certains participants ne sont pas visibles à l'écran, ou sont mal distribués par rapport au cadre), plus longs deviennent ces développements préliminaires. $\mathrm{Au}$ point d'ailleurs que certaines de ces séquences deviennent reconnaissables comme des activités propres, dont la signification doit être précisée aux nouveaux participants. Lors d'une audience particulièrement complexe, le président avertit trois visiteurs du Conseil d'État, avant la connexion, qu'« il y a toujours un petit moment de pré-audience euh de salutations ». 

fermée par son président alors qu'il ne l'a pas encore ouverte. Dans cette audience, au
moment où le président lève l'audience, il vient d'accomplir avec les autres participants
une de ces séquences préparatoires où il s'est adressé successivement à chacun d'entre eux pour initier un mode d'engagement et un positionnement adéquats. En ce sens, les participants sont conjointement arrivés au point où la situation d'audience est visiblement presque installée, où ils sont publiquement alignés par rapport à cette activité, et où il ne reste plus qu'à énoncer formellement son ouverture pour que ce soit fait. L'acte de langage rituel peut à ce stade n'apparaître que comme la dernière étape d'un long travail collectif préliminaire. Dit autrement, le fait que ce travail préparatoire soit si graduel, rend moins saillante l'action accomplie par le performatif au moment de son énonciation. Si un problème survient à ce moment, comme c'est le cas dans cet exemple puisque le magistrat et le greffier s'aperçoivent qu'il manque une pièce, le président peut lever l'audience sans que le fait qu'il ne l'a pas officiellement ouverte ne suscite aucune remarque, puisque tous les participants sont "presque " complètement engagés dans la situation d'audience.

\section{Le problème de la saillance relative des énoncés performatifs de clôture}

46 Les ressources qui permettent d'effectuer ce travail interactionnel préliminaire rendent également la situation d'audience particulièrement vulnérable à des perturbations au moment de la clôture. En effet, puisque les participants s'orientent vers le fait qu'être ensemble à l'écran, c'est être engagé dans une communauté de disponibilité par rapport à l'activité en cours, le fait de quitter l'écran est habituellement interprété comme une forme de désengagement.

Dans un des cas observés, juste après que le président ait levé l'audience, un avocat se lève et commence à sortir de l'écran. Le président le rappelle alors de la voix pour lui signaler qu'il doit rester pour signer des papiers. Lorsque je lui fais revoir cette petite séquence vidéo, l'avocat déclare qu'il a réagi automatiquement, de manière incorporée, à l'acte de langage clôturant l'audience, en se levant et partant. Il pensait pour sa part régler les papiers plus tard, puisqu'à Saint-Pierre et Miquelon, tout est petit et que ce genre de tâche s'effectue facilement au greffe. Du point de vue du président, voyant l'avocat s'apprêtant à quitter l'écran, il en conclut au contraire que celui-ci va se trouver indisponible pour tous ces arrangements, et il réagit en le rappelant à l'écran.

Études de communication, 29 | 2006 
48 deux traitements de l'énoncé performatif par lequel le président lève l'audience: se désengager de l'activité d'audience proprement dite, mais rester à l'écran, ou au contraire se désengager plus radicalement en quittant l'écran. Ceci explique pourquoi certains présidents, avant de lever l'audience, et après les débats, délibérés ou rendus de décision, initient différentes séquences visant à prendre des nouvelles, mettre au point des rendezvous, etc. Ils traitent la visibilité des participants à l'écran comme une forme de disponibilité générique, pertinente pour d'autres actions que l'audience, et anticipent à l'inverse les sorties d'écran comme des marques d'indisponibilité. D'où le souci d'exploiter la présence à l'écran des participants avant de leur fournir une opportunité de disparaître en levant l'audience.

L'étude des conversations ordinaires a montré que proposer des arrangements relatifs à des coordinations futures constituait une manière conventionnelle d'initier une préclôture de la conversation en cours (Schegloff, 1973). Les participants à l'audience à distance, sont donc susceptibles, du fait de leurs compétences conversationnelles ordinaires, de reconnaître de telles séquences, placées après que l'affaire faisant l'objet de l'audience ait été ostensiblement traitée, mais avant que l'audience soit formellement levée, comme des formes de pré-clôture. On peut donc considérer, comme pour l'ouverture, que cela conduise à des situations de désengagement graduel par rapport à la situation d'audience, avec une succession de pré-clôture où la suivante est de portée plus générale que celle qui précède. Du fait du caractère progressif de ce désengagement, l'énoncé performatif qui lève l'audience paraît à nouveau moins saillant par rapport à ce qui précède. Puisqu'on est en train de préparer des rendez vous, on n'est déjà plus tout à fait dans l'audience ou l'activité de production judiciaire au sens strict. On comprend qu'il puisse alors arriver à un président d'omettre de lever l'audience sans susciter de réactions de participants qui sont déjà alignés vers sa clôture.

\section{Conclusion}

L'efficacité performative des ouvertures et des levées d'audience doit être analysée en regard de la manière dont les participants accomplissent méthodiquement une transition entre une situation "ordinaire» et une situation de parole publique reconnaissable comme une audience judiciaire. L'accomplissement performatif sera à la fois d'autant plus heureux et net que la situation en cours réunit deux caractéristiques: l'établissement rapide, si possible à travers une simple séquence de sommation/ alignement d'une situation de parole publique, l'intelligibilité de la situation ainsi accomplie par rapport au type d'activité auquel réfère explicitement le performatif. En revanche, si, comme c'est le cas des audiences à distance, les transactions sont graduelles, et si elles procèdent d'un effort collaboratif accompli par les participants pour façonner un contexte pertinent pour l'ouverture de l'audience, le performatif apparaît comme le couronnement d'un travail interactionnel presque entièrement accompli en amont de son énonciation. Plus ce travail préparatoire est long et progressif, moins l'énoncé performatif qui le conclut apparaît saillant, tranchant et par conséquent nécessaire, ce qui explique qu'il puisse être parfois omis. Comme le suggérait Austin, pour comprendre ce qu'accomplit l'acte de langage, il faut saisir « l'acte de discours total dans la situation de discours totale $»$.

Études de communication, 29 | 2006 
51 On pourrait être tenté d'affirmer que dans les audiences à distance, les actes de langage s'affaiblissent et voient leur magie performative se ternir par rapport aux audiences en prétoire. Les caractéristiques des dispositifs de visio-conférence pourraient alors apparaître comme des causes de cet étiolement de l'efficacité performative. Une telle hypothèse constitue une réduction abusive et relève d'une forme de déterminisme technique. Rien n'empêche en effet d'imaginer que soit possible un travail de cadrage spécifique aux audiences à distance, par exemple en mettant en place de nouvelles conventions au niveau de l'arrangement des salles, des prescriptions relatives à la position à l'écran des participants, ou en accompagnant l'émergence de nouvelles règles (par exemple en ce qui concerne le placement séquentiel de la mise en connexion, ou sur la manière de préparer à l'avance celle-ci), éventuellement appuyées sur des textes juridiques. Les acteurs des deux tribunaux impliqués réfléchissent d'ailleurs en ce sens. Un tel effort de cadrage en amont des audiences à distance serait susceptible de renverser la tendance, et de faire que les audiences soient le plus souvent ouvertes selon une procédure analogue à la séquence canonique d'ouverture d'audience en prétoire, et que les audiences prêtant à des développement séquentiels entre sommation et ouverture soient beaucoup moins fréquentes. Dans ce cas, l'acte de langage ouvrant l'audience à distance retrouverait tout son lustre performatif.

52 Si le dispositif technique joue un rôle, c'est toujours à travers la médiation de l'activité où il est utilisé. La question devient celle de la capacité des acteurs impliqués dans les audiences à distance de tisser toutes les associations nécessaires pour construire le réseau socio-technico-juridique susceptible de stabiliser un tel cadre. Conformément aux théories de l'acteur réseau, ceci suppose un important travail de qualification et de traduction (Latour, 2006). Tant que ces audiences à distance ne sont traitées dans l'organisation que comme une solution exceptionnelle pour un problème exceptionnel, unique à la collectivité territoriale de Saint-Pierre et Miquelon, il est possible de douter de la capacité des promoteurs de ce type de dispositif à construire le réseau nécessaire à un tel cadrage des audiences à distance. Si par contre émerge de différents efforts au sein de l'administration judiciaire un réseau d'associations permettant de requalifier légitimement leur pratique en expérimentation susceptible d'être généralisée, et conduisant à une généralisation effective de ce dispositif à d'autres types d'audience et de tribunaux, on peut croire qu'il sera possible d'y puiser les ressources pour stabiliser un rituel d'ouverture et de levée des audience par visio-conférence dans lequel les actes de langage retrouvent saillance et efficacité performative.

Même dans ce cas, le dispositif de visioconférence continuera à jouer un autre type de rôle, intrinsèque à la situation. Ses caractéristiques et la manière dont il est mobilisé en situation façonnent les contingences susceptibles de se manifester (du fait de la fragilité du cadre de participation ou des asymétries interactionnelles qui caractérisent la visioconférence en général), les exigences normatives par rapport auxquelles les participants s'orientent (être à l'écran c'est être disponible, le tableau composé à l'écran doit être intelligible par rapport à l'activité en cours) et les ressources dont disposent localement les participants (traiter la mise en connexion comme sommation, s'adresser successivement aux participants visibles de manière ajustée à la production d'un cadre de participation pertinent, rendre visible des transitions entre différents mode de couplage, comme ceux où l'écran est transparent à l'activité et ceux où des participants plus dégagés le lisent comme un tableau). 


\section{BIBLIOGRAPHIE}

Austin, J. L., (1970), Quand dire c'est faire, Paris, Édition du seuil.

Bourdieu, P., (1982), Ce que parler veut dire. L'économie des échanges linguistiques, Paris, Fayard.

Butler, J., (1997), Excitable Speech, London, Routledge.

Chalmers, M., (2004), « A Historical View of Context », Journal of CSCW 13(3), pp. 233-224.

De Fornel, M., (1994), « Le cadre interactionnel de l'échange visiophonique », Réseaux 64, pp. 107-132.

Dodier, N., (1993), « Les appuis conventionnels de l'action. Éléments de pragmatique sociologique ", Réseaux 62, pp. 63-85.

Dourish, P., (2004), Where the Action is. The foundations of Embodied Interaction, Cambridge, M.I.T. Press.

Dreyfus, H., (1991), Being-in-the-World, A Commentary on Heidegger's Being and Time, Division I, Cambridge, M.I.T. Press.

Drew, P. et Atkinson, J. M., (1979), Order in Court. The organization of Verbal Interactions in Judicial Settings, London, Mac Millan.

Garapon, A., (2001), Bien juger. Essai sur le rituel judiciaire, Paris, Odile Jacob.

Heath, C. et Luff, P., (1992), « Media Space and Communicative Asymmetries. Preliminary Observations of Video Mediated Interactions », Human Computer Interaction 7, pp. 315-346.

Jefferson, G. et Schenkein, J., (1978), Some Sequential Negotiations in Conversation. Unexpanded and expanded versions of projected action sequences. Studies on the organization of conversational interaction, J. N. Schenkein, New York, Academic Press, pp. 155-172.

Kendon, A., (1992), « The negotiation of context in face-to-face interaction », in : A. Duranti et Goodwin C. Cambridge (eds.), Rethinking Context : Language as an Interactive Phenomenon, Cambridge University Press, pp. 323-334.

Laugier, S., (2004), « Le langage comme pratique et comme performance », in : S. Haber (ed.), L'action en philosophie contemporaine, Paris, Ellipses, pp. 191-207.

Levinson, S., (1983), Pragmatics, Cambridge, Cambridge University Press.

Relieu, M. et Brock, F., (1995), « L'infrastructure conversationnelle de la parole publique. L'analyse des réunions politiques et des interviews télédiffusées », Politix 31, pp. 77-112.

Sacks, H., Schegloff, E. et Jefferson, G., (1974), « A simplest systematics for the organization of turn-taking for conversation », Language 50(4), pp. 696-735.

Schegloff, E., \& Sacks, H., (1973), « Opening up Closings », Semiotica VIII(4), pp. 289-327. 


\section{NOTES}

1. Le travail présenté fait partie d'un projet plus global sur la justice et les nouvelles technologies, financé dans le cadre de l'ACI interdisciplinaire "terrains, techniques, théories». L'auteur remercie tout particulièrement Laurence Dumoulin, Christian Mouhanna et Jean-Claude Thoenig pour leurs commentaires et leurs critiques.

2. Pour une approche pragmatique de la force performative associée à certains écrits, voir Fraenkel dans ce numéro.

3. Le décret de 1998 qui fournit le cadre juridique pour les audiences à distance de Saint Pierre et Miquelon, indique qu'en cas d'empêchement des magistrats saint-pierrais des magistrats peuvent se substituer à eux. Dans le cas où les magistrats parisiens ne peuvent se rendre à Saint-Pierre et Miquelon, des magistrats volontaires de la Cour d'Appel de Paris peuvent se substituer aux magistrats locaux, selon un principe de double subsidiarité.

4. Sur les pré-requêtes, voir Levinson (1983).

5. Pour une présentation pédagogique des thèses heideggeriennes, et de la distinction entre « availableness » et « occurentness », voir l'ouvrage d'Hubert Dreyfus (1991).

6. Ce point est d'autant plus important qu'une tradition récente de design technologique s'oriente vers la nécessité de concevoir les artefacts de manière à ce qu'ils soient le plus possible transparents par rapport aux différentes activités dans lesquelles ils sont mobilisés (Dourish, 2004). Il semble bien qu'il faille considérer que la possibilité pour les utilisateurs d'alterner de manière visible entre différents modes de couplage constitue une importante ressource pratique, et qui doive être prise en compte dans les orientations de conception (Chalmers, 2004).

\section{RÉSUMÉS}

L'article repose la question des effets des actes de langage performatif en analysant la différence entre la manière traditionnelle d'ouvrir une audience judiciaire en présence, et ce qui se passe dans des contextes d'audiences par visioconférence. Il montre, à partir de l'analyse de données vidéo recueillies in situ comment les participants à une audience judiciaire à distance manifestent trois orientations normatives : traiter l'établissement de la connexion comme une sommation; traiter les participants visibles à l'écran comme pertinents et disponibles pour l'activité en cours; composer à l'écran un tableau intelligible comme une instantiation particulière du type d'activité en cours. Ceci permet de comprendre que puissent se développer après la connexion des séquences interactionnelles qui accomplissent graduellement la situation d'audience, au point que l'acte de langage qui ouvre l'audience apparaisse moins saillant, et soit même omis en certaines occasions. Le sens ou les effets de l'acte de langage, même pour un énoncé performatif aussi ritualisé que celui-ci, dépendent du rapport indexical que sa production entretient avec le cours d'activité dans lequel il est encastré.

This paper revisits the question of the performative effects of speech acts by analyzing empirically the differences between the openings of courtroom trials in co-present situations or by video-conferences. Using video data gathered $<i>i n$ situ $</ i>$, it shows how the participants in a court session by video-conference are oriented toward three normative issues: treating the 
creation of the audio/video link as a summons, making inferences based on the visibility (or invisibility) of distant onscreen participants regarding their availability (for interaction) and their relevance (for ongoing activity), and creating an on-screen image so as to allow this image to be grasped as a representation that makes the ongoing activity immediately accountable. These particularities of courtroom trials by video-conference explain why extended sequences of talk-in-interaction may develop between the creation of the audio link and the actual production of the ritualized opening speech act that gradually turn the situation into a recognizable courtroom setting, to the point where the ritual opening may even be omitted. The meaning and the effects of a speech act, even one as formalized and ritualized as this, always depend on the indexical relationship between the conditions of its production, the activity, and the setting in which it is embedded.

\section{INDEX}

Mots-clés : justice, NTIC

Keywords : justice, NTIC

\section{AUTEUR}

\section{CHRISTIAN LICOPPE}

Christian Licoppe est professeur de sociologie à l'École Nationale Supérieur des

Télécommunications. Ses recherches portent sur les usages des technologies de l'information et de la communication dans différents domaines : loisirs, vie privée, relation de service, professions juridiques... Elles ont en commun d'appréhender les usages en situation, afin de comprendre comment ces différents dispositifs techniques prennent place dans l'écologie de l'action. Adresse électronique : christian.licoppe@enst.fr. 